\title{
Detecting lonospheric Irregularities using Empirical Mode Decomposition of TEC for IRNSS Signals, at SVNIT, Surat, India
}

\author{
Sonal Parmar \\ ${ }^{1}$ Department of Electronics and \\ ${ }^{2}$ Telecommunication Engg. \\ ${ }^{1}$ SVNIT, Surat, ${ }^{2}$ M.P.S.T.M.E. \\ NMIMS University ${ }^{2}$ Mumbai,
}

\author{
Upena Dalal, $\mathrm{PhD}$ \\ Department of Electronics \\ Engg. SVNIT, Ichchhanath, \\ Surat 395007 Gujarat, India
}

\author{
K. N. Pathak, PhD \\ Department of Applied Physics, \\ SVNIT, Surat, India
}

\begin{abstract}
Ionospheric scintillation effects on satellite signals is one of the major source of errors which has to be determined. Severe ionospheric scintillation causes amplitude scintillations, phase scintillations, drop in carrier to noise power ratio that may results into loss of lock of the global positioning system (GPS) receiver with a particular satellite and may interrupt various navigational and positioning services. The present study comprises of measurement of ionospheric irregularities and their effect on new Indian Regional Navigation Satellite System (IRNSS). IRNSS is India's Navigation system similar to GPS which will provide various navigational and positioning services in Indian region. One of the IRNSS receiver is given by Indian Space Research Organization (ISRO), Ahmedabad, India to Department of Electronics Engineering, SVNIT, Surat which is located at low latitude region of India at Latitude $21^{\circ} 9^{\prime} 50.19 \mathrm{~N}$, and longitude $72^{\circ}$ $47^{\prime} 1.15 \mathrm{E}$. Measurement of various Ionospheric scintillation parameters like Total electron content (TEC), vertical total electron content (VTEC), carrier to noise power ratio $(\mathrm{C} / \mathrm{N})$, elevation angle of various IRNSS satellites from IRNSS 1A to 1D is presented here. TEC Variations and detection of ionospheric scintillation instances are carried out by using Wavelet based and empirical mode decomposition analysis of captured data on $23^{\text {rd }}$ September 2015 which is September Equinox period. Empirical mode decomposition decomposes signal into intrinsic mode functions (IMF) for detecting ionospheric irregularities instances using TEC data for various events like earthquake, solar storms, solar eclipse, magnetic storms, plasma bubbles etc.
\end{abstract}

\section{General Terms}

Daubechies wavelet decomposition, GPS, Hilbert Huang decomposition, IRNSS, Ionospheric irregularities, Satellite communications and wavelet based decomposition.

\section{Keywords}

Hilbert Huang transforms and Empirical mode decomposition, Ionospheric Scintillations, IRNSS signals, Total electron content, Vertical Total electron content, Wavelet analysis.

\section{INTRODUCTION}

Ionosphere layer in the atmosphere is the region which comprises of photo-ionization that creates plasma of enough concentration to have substantial impact on dynamics of this region. The spatial (with respect to geographical location) and temporal (as per the time, seasons and solar cycle) variations of ionosphere have significant effect on radio signals while propagating through it. Thus it is important to study the ionospheric influence on GPS signals in different weather circumstances at different geographical locations as mentioned by [1]. Ionospheric scintillation results into signal refraction and diffraction. GPS signal experiences advance in phase of the signals and ionospheric delay because of the free electrons present in the propagation paths. If the phase variations are large it can affect the phase locked loops in receiver section. Norsuzila et al., (2010) have done analysis for calculation of TEC and to estimate positioning error in [2].

Kintner et al., (2009) in [3] has stated that GPS signal experiences group delay and phase advance as it interacts with free electrons along its propagation path. The number of free electrons in a rectangular solid with a one square meter cross section ranging from the receiver to the satellite is known as total electron content (TEC).

The present research discusses ionospheric scintillation effects on IRNSS signals whose all satellites starting from IRNSS 1A to $1 \mathrm{~F}$ (Pseudo random number (PRN) numbers 1 to 7 ) have been launched by ISRO, India. The IRNSS system comprises of three segments namely space segment, control segment and user segment like GPS [4]. The space segment consists of IRNSS constellation of seven satellites-three in Geostationary earth orbit $(\mathrm{GEO})$ orbit $\left(\right.$ at $34^{\circ} \mathrm{E}, 83^{\circ} \mathrm{E}$, and $\left.131.5^{\circ} \mathrm{E}\right)$ and four in Geo synchronous orbit (GSO) orbit inclined at 29 degrees to the equatorial plane with their longitude crossings at $55^{\circ} \mathrm{E}$ and $111.5^{\circ} \mathrm{E}$ two in each plane as shown in figure 1.

The user can receive signals from various satellites throughout for 24 hours a day. The control segment are located at earth stations which continuously monitors and tracks various satellites locations in respective orbit and measure orbital parameters. If there is any variations in satellite location in orbit then corrections are to be made for bringing back satellites in their original position in orbits. The user segment consists of IRNSS receivers which can receive signals from various satellites decode them and give useful information for providing satellite orbital and clock information, navigational and positioning information. IRNSS receivers can be designed to receive single or dual frequency L5 Band (1176.45 MHz) or $\mathrm{S}$ band $(2492.028 \mathrm{MHz})$ which are modulated using Binary phase shift keying (BPSK) modulation technique or Binary offset carrier (BOC) technique. 


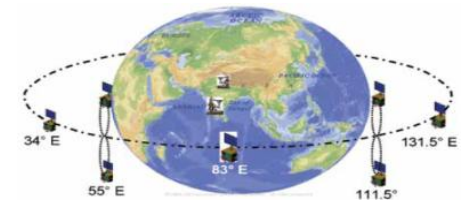

Fig 1: IRNSS Constellation indicating space segment

The experimental setup of IRNSS receiver hardware along with its software is as shown in figure 2 and space segment of IRNSS is indicated in figure 3 on $23^{\text {rd }}$ September 2015. In figure 3 satellite location is indicated in various orbits with respect to IRNSS receiver location as center of the sky plot or sky view. Satellite location are highlighted in red spots in terms of azimuth and elevation angle in degrees of various satellites PRN 1 to PRN 4. The vertical axis is comprising of elevation angle in degrees and circular outer scale is consisting of azimuth angle in degrees.

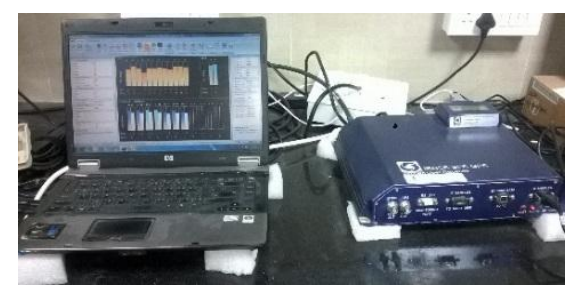

Fig 2 : IRNSS receiver lab set up at SVNIT, Surat, India.

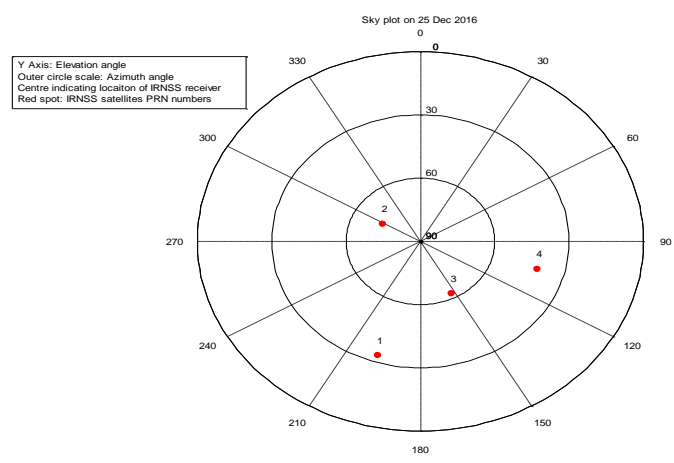

Fig 3: Space segment of IRNSS (PRN 1-4)

Table 1. Sample Satellite parameters on $23^{\text {rd }}$ Sept. 2015

\begin{tabular}{|l|l|l|l|}
\hline $\begin{array}{l}\text { Sr. } \\
\text { No. }\end{array}$ & $\begin{array}{l}\text { PRN } \\
\text { number } \\
\text { of } \\
\text { satellites }\end{array}$ & $\begin{array}{l}\text { Azimuth } \\
\text { angle } \\
\text { (degrees) }\end{array}$ & $\begin{array}{l}\text { Elevation } \\
\text { angle } \\
\text { (degrees) }\end{array}$ \\
\hline 1 & 1 & 197.93 & 33.38 \\
\hline 2 & 2 & 299.31 & 72.25 \\
\hline 3 & 3 & 153.27 & 62.79 \\
\hline 4 & 4 & 105.39 & 41.39 \\
\hline
\end{tabular}

In this paper the ionospheric scintillation parameters measurement is done on $23^{\text {rd }}$ September 2015 data which is a September equinox period, it is the moment when the Sun appears to cross the celestial equator, heading southward.
Section 2 is discussing related work. Section 3 is discussing analysis and results. Finally the conclusion is presented in section 4 .

\section{RELATED WORK REVIEW}

Ionospheric scintillation is the most effective variable on various global navigation satellite systems (GNSSs) signals. In satellite communication applications, the scintillation effects produces rapid fluctuations on the propagated satellite signals and causes positioning error. Since Surat is located in low latitude region, scintillation effects on satellite signals are to be analyzed. Very few researchers are working on IRNSS signals. Similar research on ionospheric effects on GPS signals have been done earlier.

Raghu et al., (2016) has presented work for determining about the availability, visibility and position accuracies of IRNSS in [4]. The evaluation of the coordinates and root mean square (rms) errors are calculated for all three GNSS systems. Thoelert et al., (2014) in [5] has discussed tracking and the measurements are used to depict the short-term clock stability of IRNSS-1A satellite. A tool has been developed for interference analysis of the various GNSS signals to analyse the degradation of the IRNSS signals due to troposphere, ionosphere and multipath effects by Mishra et al., (2016) in [6]. Mishra et al (2015) have estimated navigation parameter through software and compared the parameters of existing GNSS signal with IRNSS signals in [7]. Zaminpardaz et al., (2016) have given understanding into IRNSS for determining its positioning performance using L5 psuedorange tracking data. This analysis was one of the first results of the IRNSS stand-alone positioning capabilities over Australian region in [8].

The models of receiver orbit propagation algorithms with proposed ephemeris are informed and are compared with standalone GLONASS model have been developed by Chandrasekhar et al., (2015) in [9]. Babu et al., (2012) have determined offset for the IRNSS satellite clocks useful for launching of IRNSS satellites in [10].

Ahmed et. al., (2015) have presented improved phase scintillation index using the wavelet based filtering technique to determine the scintillation effects at European high latitudes in [11]. The improved phase scintillation is then compared with the original phase scintillation index. Sauli et. al., (2006) had proposed a wavelet-based detection and characterisation of the propagation of acoustic-gravity waves induced in the ionosphere during the solar eclipse of August 11, 1999 in [12]. Fernandez et. al., (2013) had computed a parameter which estimates the local ionospheric response to a geospheric event which triggers an ionospheric storm in [13].

Akhoondzadeh (2012) have utilized various methods for TEC variations during Tohoku earthquake in [14]. Senanayake (2013) has emphasized on positive impacts of various Indian Navigational Satellite programmes for the civilian applications over Sri Lankan region in [15]. Grinsted et, al., (2004) have utilized cross wavelet transform and wavelet coherence for determining relationships in time frequency space between two time series in [16]. Ratnam et, al., (2015) have demonstrated use of GPS Ionospheric Scintillation TEC monitor and receiver which is located at the low latitude station, K. L. University, India in [17]. Hobiger and schuh has derived ionospheric parameters by Very Long Baseline 
Interferometry (VLBI), Topex/Poseidon and GPS in [18]. The wavelet scalograms are also used.

Huang and $\mathrm{Wu}$ (2008) had presented results which showed that empirical mode (EMD) Hilbert-Huang transform (HHT) decomposition and Hilbert spectral analysis are adaptive data analysis method for various geophysical research in [19]. Krankowski et, al., (2005) had given an extensive database of Total Electron Content (TEC) measurements which has become available from both ground- and space-based observations in [20]. Lei et, al., (2008) have presented 9 and 7 day periodic oscillations in the global mean TEC from 1 January 2005 to 31 December 2006 in [21].

Thus in this section review of work done for analysis of ionospheric parameters using wavelet analysis and Hilbert Huang transform (HHT) is presented. In the following section the introduction to various ionospheric scintillation parameters like total electron content, Vertical Total electron content (VTEC), Carrier to noise power ratio and elevation angle is presented.

\section{IONOSPHERIC SCINTILLATION MEASUREMENT PARAMETERS}

\subsection{Total electron content (TEC)}

TEC is an important parameter in the Ionospheric studies and for the correction of Ionospheric effects which affects performances of various Global Navigation Satellite System (GNSS) positioning accuracy as mentioned by Misra and Enge (2001) in [22] and Tanna et al., in (2013) in [23]. It is an important component of the space weather for both tropical and subtropical.

A dual-frequency GPS receiver measures the difference in ionospheric delay between the L1 and L2 satellite signals. TEC can is calculated using following equation (1) [24, 25]:

$$
T E C=\frac{1}{40.3}\left(\frac{f_{1}^{2} * f_{2}^{2}}{f_{2}^{2}-f_{1}^{2}}\right)\left(P_{1}-P_{2}\right)
$$

Where in equation (1), $\mathrm{f}_{1}=2492.028 \mathrm{MHz}$ (L5 band frequency) and $\mathrm{f}_{2}=1176.45 \mathrm{MHz}$ ( $\mathrm{S} 1$ band frequency) $\mathrm{P} 1$ and $\mathrm{P} 2$ are Pseudo ranges of the frequencies L5 and S band respectively.

\subsection{Elevation angle (EIv)}

In the present work, measurements with a satellite's elevation angle greater than 30 degrees are taken into account in order to minimize the effects of multipath on the observations. Elevation angle in Radians = Elevation angle in degrees $*$ (Pi/180).

\subsection{Vertical TEC}

The TEC varies smoothly from day to night as dayside atmosphere of the Earth is ionized by the Sun's extreme ultraviolet radiation. During a plasma bubble, the F2 layer of the ionosphere will become unstable and may even disappear. In this region the total electron content is in the highest density in range $10^{16}$ to $10^{19}$ with minimum and maximum occurring at midnight and mid-afternoon approximately.
Earlier studies state that the plasma bubble usually occurs after the sunset period when the TEC fluctuates and reduces about 5TECU to 20TECU. Vertical TEC is calculated using equation 2 [2]: $\operatorname{VerticalTEC}($ VTEC $)=S T E C * \cos \left[\arcsin \left(\frac{R_{e} \cos \theta}{R_{e}+h_{\max }}\right)\right]$

Where Radius of Earth, $\mathrm{Re}=6378 \mathrm{Km}$, Height of Ionospheric shell, $\mathrm{h}_{\max }=350 \mathrm{Km}, \Theta=$ elevations angle in radians at the ground station.

\subsection{Carrier to noise power ratio}

Carrier to noise power ratio ( $\mathrm{C} / \mathrm{N}$ ratio) is also a measure to predict ionospheric scintillation on IRNSS signals. It is measured in decibels. If $\mathrm{C} / \mathrm{N}$ ratio is less than 25 or $30 \mathrm{~dB} / \mathrm{Hz}$ then it may result into loss of lock of the receiver to the particular satellite and may disrupt services offered by IRNSS or any GNSS system.

Thus the measurement and calculations of major ionospheric parameters are presented in this section. Further the results and methodology are discussed.

\section{DATA ANALYSIS AND METHODOLOGY}

Data presented here for analysis is taken from IRNSS User receiver installed in Electronics Engineering Department, SVNIT, Surat. Results presented here are utilizing MATLAB software and based on the data captured by IRNSS receiver on $23^{\text {rd }}$ September 2015 which is a September equinox period.

\subsection{Total Electron Content variation on 23 September 2015.}

Total Electron Content (TEC) Variation for satellite PRN numbers $1-4$ on $23^{\text {rd }}$ September 2015 is presented here. Pseudo Random number (PRN) numbers are the numbers given to various satellites for identifying a particular satellite. Figure 4 to 7 are illustrating variation of carrier to noise $(\mathrm{C} / \mathrm{N})$ power ratio in $\mathrm{dB}-\mathrm{Hz}$ for both $\mathrm{L} 5$ and $\mathrm{S}$ band of frequencies, Elevation angle in degrees for respective PRN satellite, TEC in TEC units (TECU) and Vertical TEC in TECU for PRN 1 to PRN 4 respectively. Normally the Peaks of TEC are during the $3 \mathrm{pm}$ to $5 \mathrm{pm}$ (IST) intervals. During the morning hours around 5 am the TEC values are quite very low. Again after sunset TEC value decreases. Normally the TEC is varying in the 0 to 80 (TECU) in this region.

\subsection{Second order Daubechies Wavelet decomposition}

A wavelet is a waveform of effectively limited duration which has an average value of zero. Wavelet analysis is the breaking up of a signal into shifted and scaled versions of the original or mother wavelet. One-dimensional analysis is based on one scaling function $\mathrm{f}$ and one wavelet. A wavelet exists for a finite duration. Wavelet analysis is similar to Fourier analysis in the sense that it breaks a signal down signal into its constituent parts for analysis.

The wavelet transform breaks the signal into its "wavelets", scaled and shifted versions of the "mother wavelet". The wavelet transform allows exceptional localization in both the time domain via translations of the mother wavelet, and in the 
scale (frequency) domain via dilations. The translation and dilation operations applied to the mother wavelet are performed to calculate the wavelet coefficients, which represent the correlation between the wavelet and a localized section of the signal. There are two concepts in wavelet: Scaling and Shifting.

\subsubsection{Scaling}

For example we have a signal $\psi(t)$. Scaling refers to the process of stretching or shrinking the signal in time which can be expressed as in equation (3):

$$
\Psi\left(\frac{t}{s}\right) s>0
$$

Where $\mathrm{s}$ is the scaling factor which is a positive value and corresponds how much a signal is scaled in time. The scale factor is inversely proportional to frequency. Scaling a sine wave by 2 results in reducing its original frequency by $1 / 2$ or an octave. For a wavelet there is a reciprocal relationship between the scale and the frequency with a constant of proportionality. This constant of proportionality is called the center frequency of the wavelet.

Mathematically the equivalent frequency is defined as in equation (4):

$$
F_{e q}=\frac{C_{f}}{s \delta_{t}}
$$

Where $\mathrm{C}_{\mathrm{f}}$ is the center frequency of the wavelet, $\mathrm{S}$ is the wavelet scale, $\delta_{t}$ is the sampling interval.

\subsubsection{Shifting}

Shifting a wavelet means delaying or advancing the onset of a wavelet along the length of a signal. A shifted wavelet represented using this notation means that the wavelet is shifted and centered at ' $\mathrm{k}$ '.

The second order Daubechies wavelet decomposition of TEC in TECU (minute's data) for PRN 1 to PRN 4 for both L5 and $\mathrm{S}$ band are indicated from figures 8 to 11 . Figure 8 represents TEC for PRN 1 for S and L5 band, Figure 9 represents TEC for PRN 2 for S and L5 band, Figure 10 represents TEC for PRN 3 for S and L5 band, and Figure 11 represents TEC for PRN 4 for S and L5 band. In figure $\mathrm{x}$-axis represents position along the signal (time), the $y$-axis represents scale, and the colour at each $\mathrm{x}-\mathrm{y}$ point represents the magnitude of the wavelet coefficient $C$. These are the coefficient plots generated by the graphical tools in Matlab. Wavelet decomposition is important for determining minor variations in TEC for determining instances of ionospheric irregularities.

\subsection{Empirical Mode Decomposition (HHT- EMD)}

The EMD works in temporal space directly rather than in the corresponding frequency space. The mixture of the eminent Hilbert spectral analysis (HAS) and the empirical mode decomposition (EMD), designated as the Hilbert-Huang transform (HHT) by NASA, represents such an exemplar shift of data investigation approach. The HHT is intended explicitly for examining nonlinear and nonstationary information. The main part of HHT is EMD with which any complex data set can be decomposed into a determinate and frequently small number of intrinsic mode functions (IMFs). The instantaneous frequency defined using the Hilbert transform denotes the physical meaning of local phase change better for IMFs than for any other non-IMF time series. This decomposition method is adaptive and so highly effectual. As the decomposition is based on the local features of the data, it is applicable to nonlinear and nonstationary processes as stated by Huang and Wu (2008) in [19].

The decomposition has implicitly a simple assumption that, at any given time, the data may have many coexisting simple oscillatory modes of significantly different frequencies, one superimposed on the other. Each component is defined as an intrinsic mode function (IMF) which satisfies the following conditions: (i) In the whole data set, the number of extrema and the number of zero crossings must either equal or differ at most by one. (ii) At any data point, the mean value of the envelope defined using the local maxima and the envelope defined using the local minima is zero.

\subsubsection{Hilbert Spectral Analysis}

The determination of the expansion of HHT is to provide an alternate view of the time-frequency-energy model of data. In this method, the nonlinearity and nonstationarity can be dealt with better than by using the traditional paradigm of constant frequency and amplitude as mentioned by Huang and $\mathrm{Wu}$ (2008) in [19]. One way to express the nonstationarity is to find instantaneous frequency and instantaneous amplitude. For any function $\mathrm{x}(\mathrm{t})$ of $\mathrm{L}^{\mathrm{p}}$ class, its Hilbert transform $\mathrm{y}(\mathrm{t})$ is as given in equation (5) [19].

$$
y(t)=\frac{1}{\pi} P \int_{-\infty}^{\infty} \frac{x(\tau)}{t-\tau} d \tau
$$

In equation (5), "P" is the Cauchy principal value of the singular integral. With the Hilbert transform y ( $t)$ of the function $x(t)$, we obtain the analytic function, as given in equation (6) and (7) [19]:

$$
\begin{aligned}
& z(t)=x(t)+i y(t)=a(t) e^{i \theta(t)} \\
& a(t)=\left(x^{2}+y^{2}\right)^{\frac{1}{2}}, \theta(t)=\tan ^{-1} \frac{y}{x}
\end{aligned}
$$

$$
\text { where }-i=\sqrt{-1}
$$

In equation (7), "a" is the instantaneous amplitude, and $\Theta$ is the instantaneous phase function. The instantaneous frequency is given in equation (8) [19]:

$$
\omega=\frac{d \theta}{d t}
$$

With both amplitude and frequency being a function of time, we can express the amplitude in terms of a function of time and frequency, $H(\omega, t)$. The marginal spectrum can then be defined as in equation (9) [19]. 
$h(\omega)=\int_{0}^{T} H(\omega, t) d t$,

In the equation (9), $[0, \mathrm{~T}]$ is the temporal domain within which the data is defined. The marginal spectrum represents the accumulated amplitude over the entire data in a probabilistic sense and offers a measure of the total amplitude (or energy) contribution from each frequency value, serving as an alternative spectrum expression of the data to the traditional Fourier spectrum. A perfect IMF, as given in equation (10) [19].

$$
x(t)=e^{\frac{-t}{256}} \sin \left[\frac{\pi t}{32}+0.3 \sin \left(\frac{\pi t}{32}\right)\right],
$$

The term on the right-hand side of equation (6) offers an apparent time-frequency-energy expression for function $\mathrm{x}(\mathrm{t})$, such as the one given by equation (10). However, for an arbitrary $x(t)$, the instantaneous frequency obtained using the above method is not necessarily physically meaningful.

\subsubsection{EMD Process}

A signal $\mathrm{x}(\mathrm{t})$ is thus divided into: the fine scale detail, $\mathbf{d}(\mathbf{t})$, and the residual, $\mathbf{m}(t)$, so $x(t)=m(t)+d(t)$. This detail, $d$ $(t)$, becomes the first IMF and the sifting process is repeated on the residual, $m(t)=x(t)-d(t)$. The sifting process requires that a local average of the function be defined. Huang's solution to finding a local average is by creating maximum and minimum envelopes around the signal using natural cubic splines through the respective local extrema. The local average is approximated as the mean of the two envelopes.

\subsubsection{EMD Algorithm}

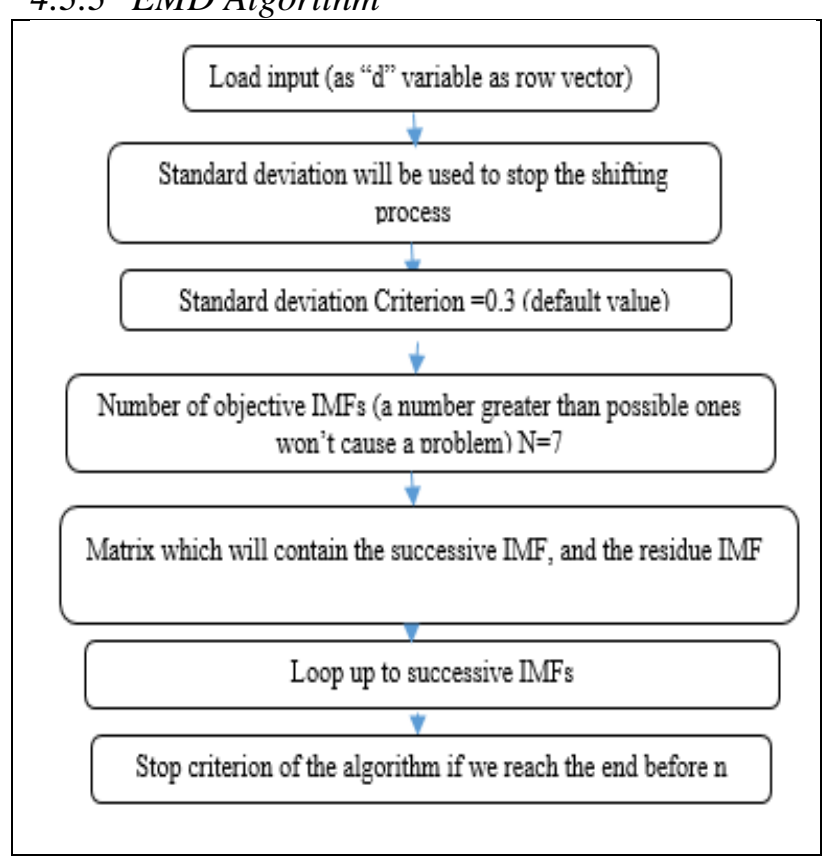

The Empirical mode decomposition of TEC for PRN 1 to PRN 4 for both L5 and S band are indicated from figures 12 to 15. Figure 12 represents Empirical mode decomposition of TEC for PRN 1 for L5 band and S band, figure 13 represents Empirical mode decomposition of TEC for PRN 2 for S band and L5 band, figure 14 represents Empirical mode decomposition of TEC for PRN 3 for S band and L5 band and figure 15 represents Empirical mode decomposition of TEC for PRN 4 for S band and L5 band.

\section{CONCLUSIONS}

The effects of the ionosphere on satellite signals used for different communication and navigation applications have been extensively investigated in the last many years. The ionosphere is known to be the major source of receiver degradation and failure for the global positioning system (GPS). It causes GPS signal's group velocity to decrease and phase velocity to advance. These effects allow the determination of the total electron content (TEC) along the signal path from the GPS satellite to the receiver. It can be seen that Hilbert Huang decomposition decomposes TEC data into minor variations which benefits in identifying ionospheric irregularities instances and finds applications in determining variations in TEC during various events like earthquake, geomagnetic storm, solar flares and plasma bubble etc.

Ionospheric scintillation measurement using TEC variations were investigated using IRNSS receiver data recorded at Electronics Engineering department low latitude station, Surat, India. To study the effect of Ionospheric scintillation on the performance of GPS receivers, the investigation will be further carried out on long time data. In general, scintillations can lead to cycle slips and loss-of-lock in GNSS receivers. Thus here wavelet based decomposition and Empirical mode decomposition (EMD) is done on various ionospheric scintillation measurement parameters. And it is found graphically that EMD is able to decompose the parameters very sharply so that the instances of ionospheric effects and ionospheric irregularities can be determined from the decomposition. HHT gives sharper results as compared to wavelets.

\section{ACKNOWLEDGMENTS}

First Author would like to thanks ISRO Ahmedabad for granting IRNSS receiver for carried out research related to ionospheric scintillation irregularities.

\section{REFERENCES}

[1] SBAS Ionospheric working group, "SBAS Ionospheric Scintillations on GNSS- A White Paper", on line document:

http://waas.stanford.edu/papers/IWG/sbas_iono_scintillat ions_white_paper, November 2010.

[2] Y. Norsuzila, M. Abdullah, M. Ibrahim, Z. Zakaria. Total Electron Content (TEC) and Estimation of Positioning Error Using Malaysia Data. World Congress on Engineering 2010; I.

[3] Kintner, P., Humphreys, T., Hinks, J., "GNSS and Ionospheric Scintillation- How to survive the next solar maximum", on line access- www.insidegnss.com, pp.2229, August 2009.

[4] Raghu, N., Manjunatha, K.N., Kiran, B., "Determination and preliminary analysis of position accuracy on IRNSS satellites", International conference on Communication and Signal Processing, p. 765-769, 2016.

[5] Thoelert, S., Montenbruck, O., Meurer, M., "IRNSS-1A; Signal and clock characterization of the Indian regional navigation system", GPS solutions, Volume 18, p.147$152,2014$. 
[6] Mishra, D., Ganesaan, R.P., Banik, A., "Study and analysis of GNSS signals using In-house developed interference tool", Communications on Applied Electronics (CAE), p.32-38, 2016.

[7] Mishra, D., Bhardwaj, S., Banik, A., Ram, T.V.S., Majithiya, P., "Navigation Signal simulator for performance analysis of GNSS signals", Communications on Applied Electronics (CAE), Volume 1, p.24-30, 2015.

[8] Zaminpardaz, S., Teunissen, P.J.G., Nadarajah, N., "IRNSS stand-alone positioning: first results in Australia", Journal of Spatial Science, Volume 61, No.1, p.5-27, 2016.

[9] Chandrasekhar, M.V., Rajarajan, D., Satyanarayana, G., Tirmal, N., Rathnakara, S.C., Ganeshan, A.S., "Modernized IRNSS broadcast ephemeris parameters", Control Theory and Informatics, Volume 5, No. 2, p. 1$10,2015$.

[10] Babu, R., Rethika, T., Rathnakara, S.C., “Onboard atomic clock frequency offset for Indian Regional Navigaiton Satellite system", International Journal of Applied Physics and Mathematics, Volume 2, p. 270272, 2012.

[11] Ahmed, A., Tiwari, R., Stangeways, H. J., Dlay, S., Johnsen, M. G., "Wavelet-based Analogous Phase Scintillation Index for High Latitudes", Space Weather, pp. 1-18, 2015.

[12] Sauli, P., Abry, P., Boska, J., Duchayne, L., “Wavelet characterisation of ionospheric acoustic and gravity waves occurring during the solar eclipse of August 11, 1999", Journal of Atmospheric and Solar-Terrestrial Physics 68, pp. 586-598, 2006.

[13] Fernandez, L. I., Meza, A. M., Zele, V. M. A., "Wavelet analysis of the ionospheric response at Mid-latitudes during the April 2000 storm using magnetograms and vTEC from GPS", GeoActa 37, Volume 2, pp. 178-196, 2013.

[14] Akhoondzadeh, M., "Anomalous TEC variations associated with the powerful Tohoku earthquake of 11 March 2011", Nat. Hazards Earth Syst. Sci., 12, 14531462, May 2012.

[15] Senanayake, I.P., "Anticipated prospects and civilian applications of Indian satellite navigation services in Sri Lanka", The Egyptian Journal of Remote Sensing and Space Science, Volume 16, Issue 1, Pages 1-10, June 2013.
[16] Grinsted, A., Moore, J. C., and Jevrejeva, S, “Application of the cross wavelet transform and wavelet coherence to geophysical time series", Nonlinear Processes Geophys., 11, 561-566, November 2004

[17] D. V. Ratnam, G. Sivavaraprasad and J. Lee, "Automatic ionospheric scintillation detector for global navigation satellite system receivers," in IET Radar, Sonar \& Navigation, vol. 9, no. 6, pp. 702-711, 2015.

[18] Hobiger, T., Schuh, H., "Comparison of vertical total electron content values obtained by different space geodetic techniques", Advanced Geodesy.

[19] Huang, Norden E., Wu, Zhaohua, “ A review on HilbertHuang transform: Method and its applications to geophysical studies", Reviews of Geophysics, Volume 46, Issue 2, pp. 1944-9208, June 2008

[20] Krankowski A., Hobiger T., Schuh H., Kosek W., Popinski W., "Wavelet analysis of TEC measurements obtained using dual frequency space and satellite techniques" in Proceedings of Journées, pp. 290-293, 2005.

[21] Lei. J., Thayer, J, P., Forbes, J, M., Wu, Q., She, C., Wan, W., Wang, W., "Ionosphere response to solar wind high-speed streams", Geophysical Research Letters, Volume 35, Issue 19, pp. 1-5, 2008.

[22] Misra, P., Enge, P., "Global Positioning System, Signals, Measurements, and Performance", Ganga Jamuna Press, 2001.

[23] Tanna, H.J., Karia, S.P., Pathak, K.N., “A study of L band scintillations during the initial phase of rising solar activity at an Indian low latitude station", Advances in Space Research, Volume 52, pp. 412-421, April 2013.

[24] S. Parmar, U. Dalal and K. N. Pathak, "Analysis of Total Electron Content using GDF and Nakagami-m Distribution for Indian Regional Navigation Satellite system (IRNSS) signals at low latitude station, Surat, India, International Union of Radio Science, URSI-RCRS

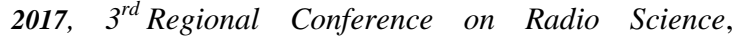
Tirupati, India, March 2017.

[25] S. Parmar, U. Dalal and K. N. Pathak, "A Study of diurnal variation of Ionospheric Scintillation effects on GPS signals at low latitude equatorial anomaly station, Surat, India," 2015 International Conference on Industrial Instrumentation and Control (ICIC), Pune, 2015, pp. 944949. 


\section{APPENDIX}

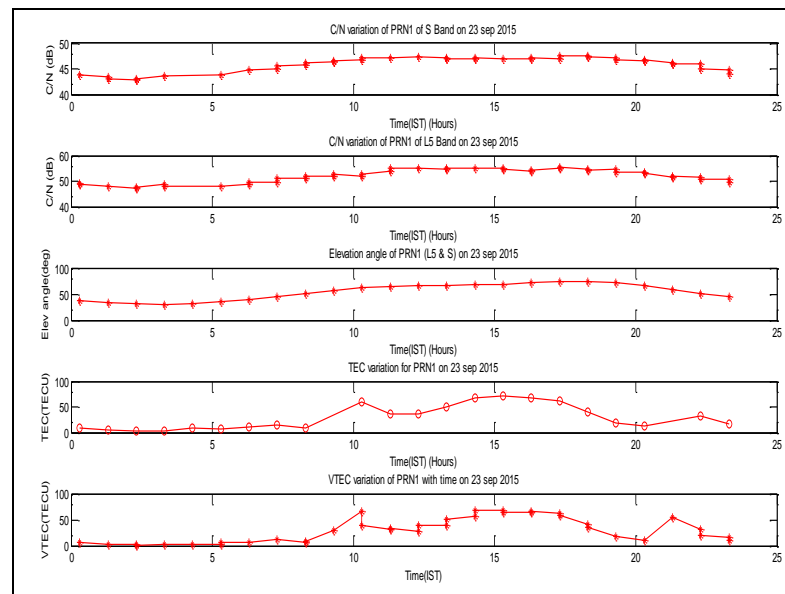

Fig 4: Variation of $\mathrm{C} / \mathrm{N}$ ratio ( $\mathrm{L5}$ and $\mathrm{S}$ band), Elevation angle, TEC and VTEC of PRN1 on 23 Sept. 2015.

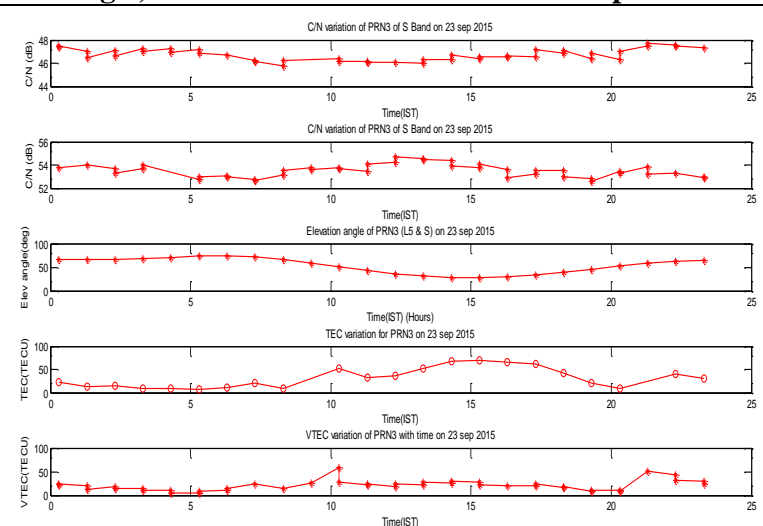

Fig 6: Variation of $\mathrm{C} / \mathrm{N}$ ratio (L5 and $\mathrm{S}$ band), Elevation angle, TEC and VTEC of PRN3 on 23 Sept. 2015.

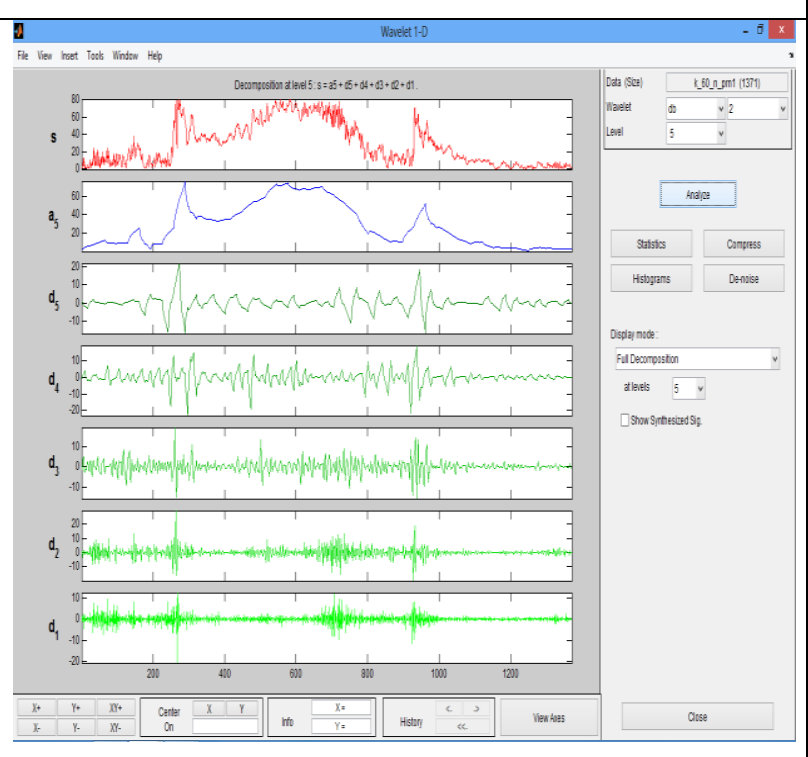

Fig 8: Second order Daubechies Wavelet decomposition Level 2 of TEC for PRN 1 (L5 and S Band)

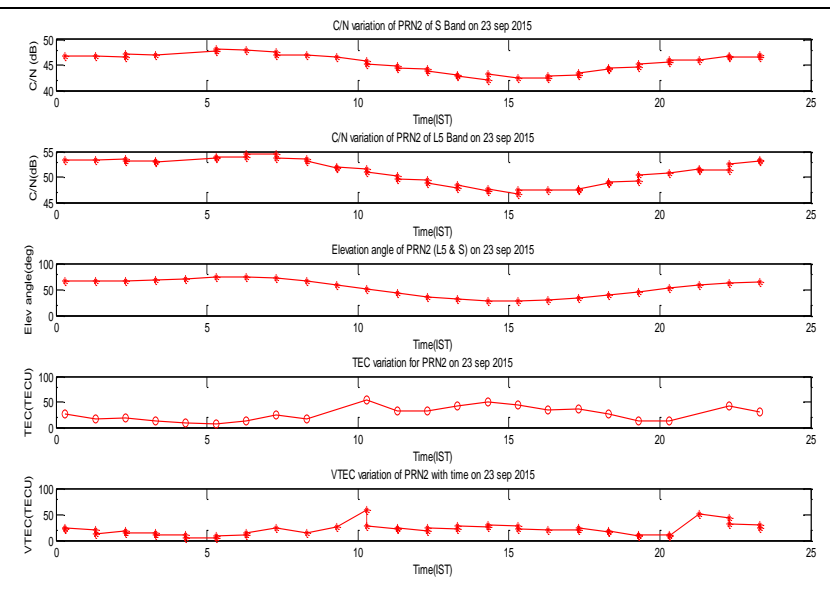

Fig 5: Variation of $\mathrm{C} / \mathrm{N}$ ratio (L5 and $\mathrm{S}$ band), Elevation angle, TEC and VTEC of PRN2 on 23 Sept. 2015.

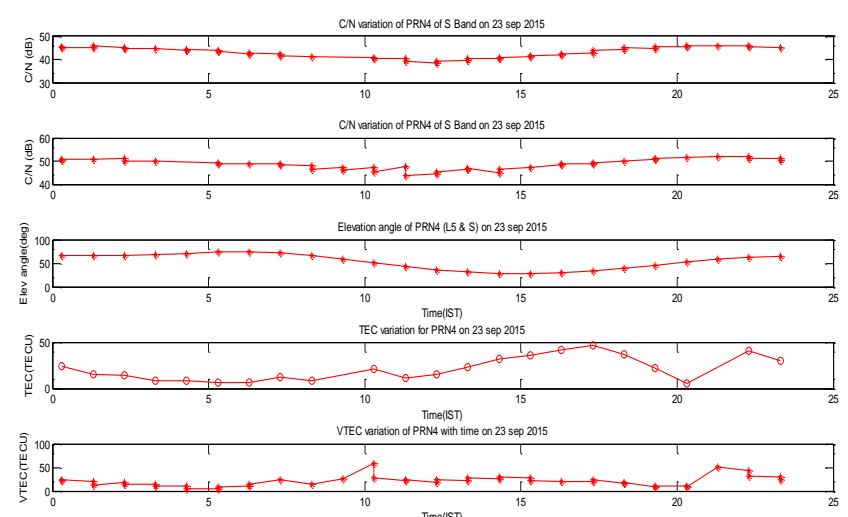

Fig 7: Variation of $\mathrm{C} / \mathrm{N}$ ratio ( $L 5$ and $\mathrm{S}$ band), Elevation angle, TEC and VTEC of PRN4 on 23 Sept. 2015.

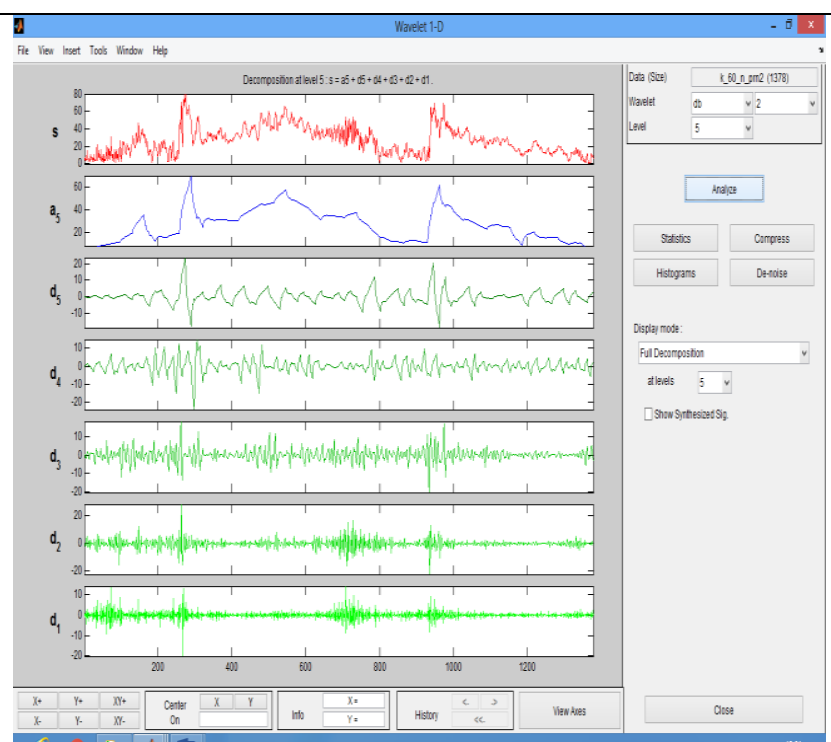

Fig 9 : Second order Daubechies Wavelet decomposition Level 2 of TEC for PRN 2 (L5 and S Band) 


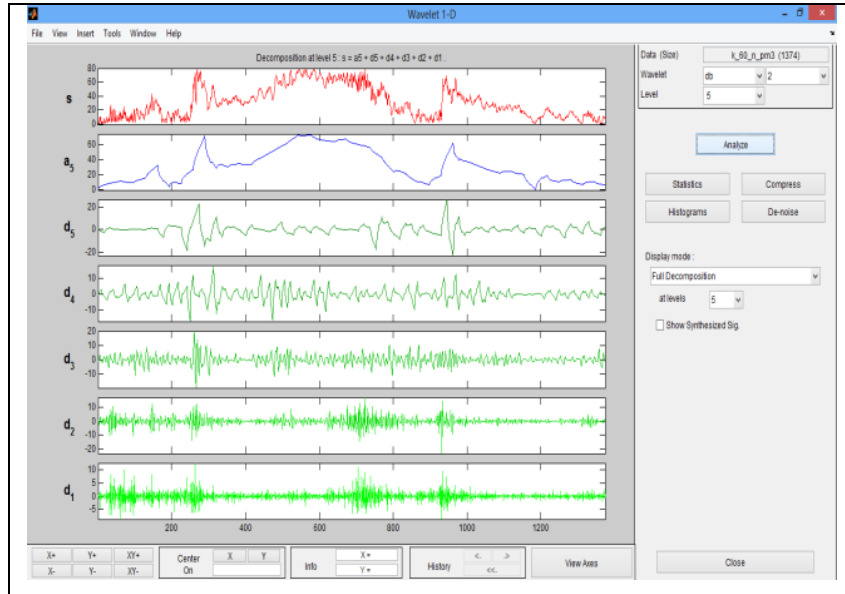

Fig 10: Second order Daubechies Wavelet decomposition Level 2 of TEC for PRN 3 (L5 and S Band)

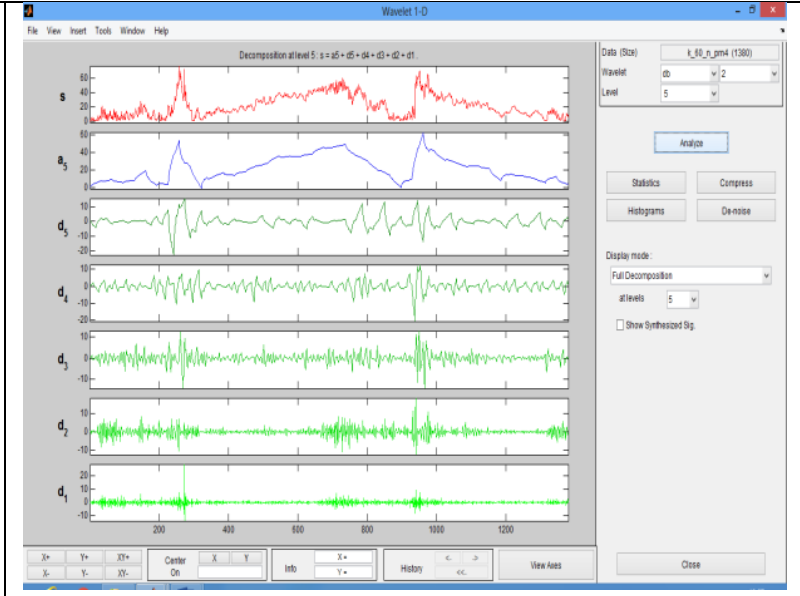

Fig 11: Second order Daubechies Wavelet decomposition Level 2 of TEC for PRN 4 (L5 and S Band)

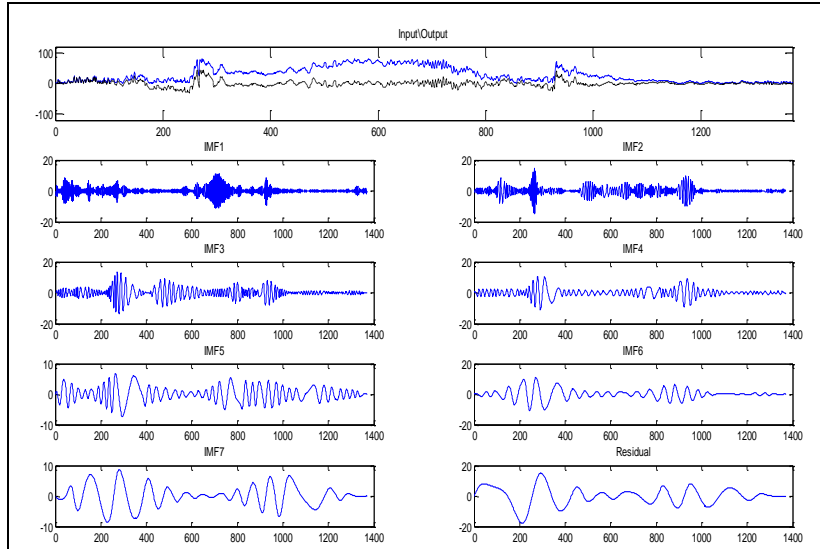

Fig 12 :Empirical mode decomposition of TEC for PRN 1 (L5 and S Band) (k_60_n_prn1 minutes data)

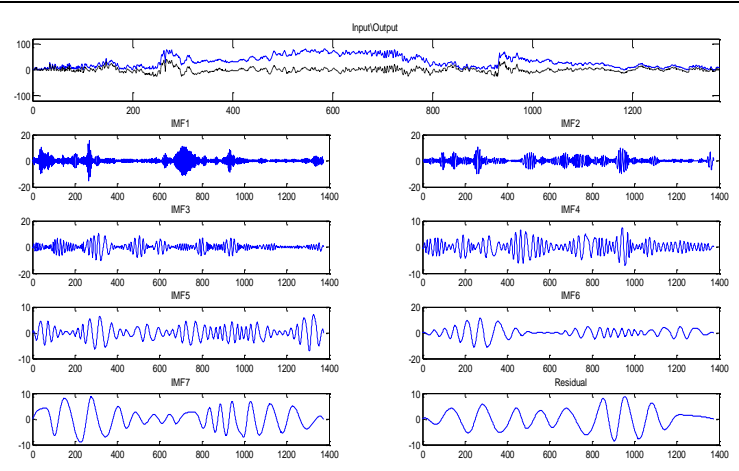

Fig 14: Empirical mode decomposition of TEC for PRN 3 (L5 and S Band) (k_60_n_prn3 minutes data)

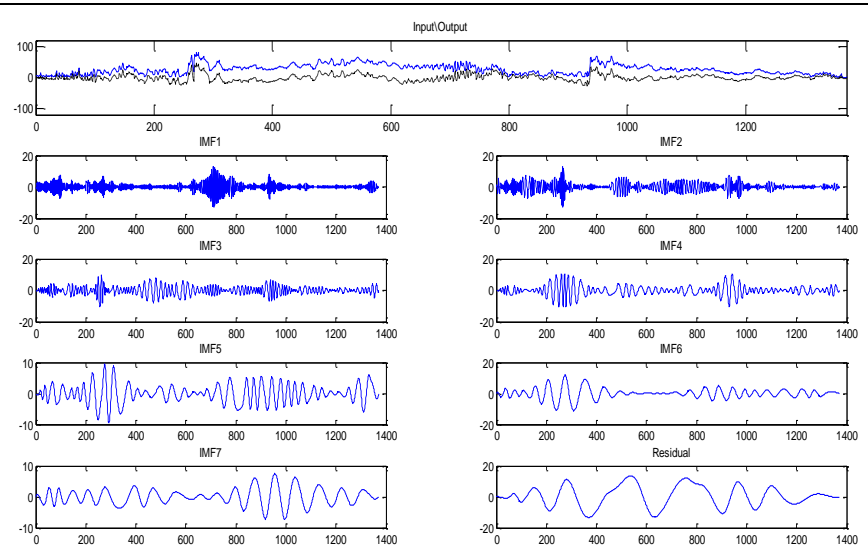

Fig 13: Empirical mode decomposition of TEC for PRN 2 (L5 and $S$ Band) (k_60_n_prn2 minutes data)

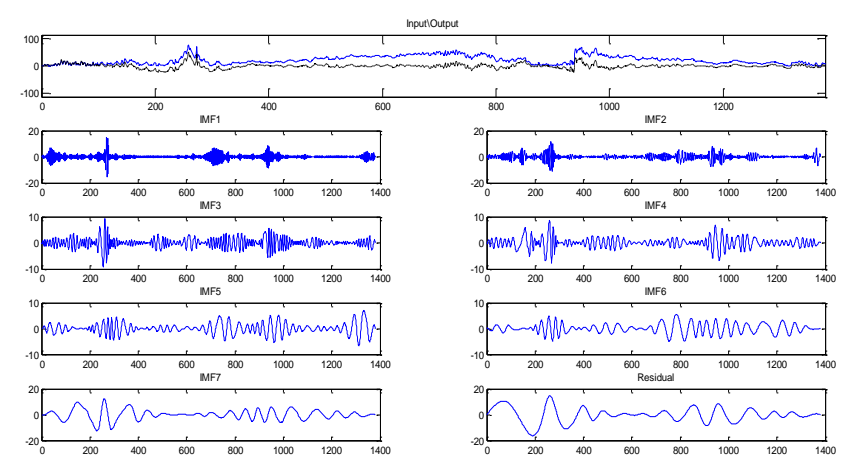

Fig 15: Empirical mode decomposition of TEC for PRN 4 (L5 and $S$ Band) (k_60_n_prn4 minutes data) 